\title{
Features of the microstrip proportional counter technology (invited)
}

\section{Budtz-Jørgensen, Carl}

\section{Published in:}

Review of Scientific Instruments

Link to article, DOI:

$10.1063 / 1.1142679$

Publication date:

1992

Document Version

Publisher's PDF, also known as Version of record

Link back to DTU Orbit

Citation (APA):

Budtz-Jørgensen, C. (1992). Features of the microstrip proportional counter technology (invited). Review of Scientific Instruments, 63(1), 648-654. https://doi.org/10.1063/1.1142679

\section{General rights}

Copyright and moral rights for the publications made accessible in the public portal are retained by the authors and/or other copyright owners and it is a condition of accessing publications that users recognise and abide by the legal requirements associated with these rights.

- Users may download and print one copy of any publication from the public portal for the purpose of private study or research.

- You may not further distribute the material or use it for any profit-making activity or commercial gain

- You may freely distribute the URL identifying the publication in the public portal

If you believe that this document breaches copyright please contact us providing details, and we will remove access to the work immediately and investigate your claim. 


\title{
Features of the microstrip proportional counter technology (invited)
}

\author{
Carl Budtz-Jørgensen \\ Danish Space Research Institute, G1. Lundtoftevej 7, DK-2800 Lyngby, Denmark
}

(Presented on 16 July 1991)

\begin{abstract}
The microstrip proportional counter (MSPC) is a new approach in gas-filled detector technology. In contrast with the classical multiwire proportional counter (MWPC) detectors, the electrodes of the MSPC are deposited by photolithographic techniques onto a rigid substrate. The technique offers many important advantages: An optimal energy resolution; The possibility of matching the electrode structure to the expected spatial resolution, i.e., smaller electrode distances than are possible for MWPCs; A much faster signal development than for the MWPC because of the small distance (few $100 \mu \mathrm{m}$ ) between the anode and cathode strips which allows the positive ion cloud created in the avalanche to be collected rapidly with reduced space charge effects and increased counting-rate capability; A rigid electrode structure which is not deformed under the action of an electric field; A relatively simple fabrication technique which allows the rapid replacement of damaged electrode structures. The applications of the MSPC within the fields of $\mathrm{x}$-ray astronomy, neutron diffraction, high energy physics, and synchrotron radiation will be discussed.
\end{abstract}

\section{INTRODUCTION}

The microstrip proportional counter (MSPC) is a new approach to gas-filled detector technology. In contrast with the classical multiwire proportional counter (MWPC) detectors, the electrodes of the MSPC are deposited by photolithographic techniques, such as are used in modern electronic-chip production, onto a rigid substrate. Strip structures can be fabricated with a precision of $0.1-0.2 \mu \mathrm{m}$ and plates with surface areas $18 \times 18 \mathrm{~cm}$ have been produced. Oed ${ }^{1}$ proposed this technique and his pioneering MSPC demonstrated gas gain uniformity and excellent energy resolution for detection of low energy light particles. The microstrip (MS) plate developed by Oed will constitute the sensor element of a large $\left(\sim 0.7 \mathrm{~m}^{2}\right)$, one-dimensional neutron detector presently under construction at Institute Laue-Langevin. ${ }^{2}$

At DSRI we adapted the MS technique for use in $x$-ray astronomy. ${ }^{3}$ When used as an $\mathrm{x}$-ray detector, the MSPC has proven to be very powerful. It has an energy resolution ${ }^{3}$ which is at least as good as that which can be achieved for a single-wire proportional counter.

The MSPC is also being developed for use in high energy physics. Two groups, Angelini et al. ${ }^{4}$ and Hartjes et al., ${ }^{5}$ working on the development of the MSPC, report excellent localization accuracies $(<50 \mu \mathrm{m})$ for minimum ionizing particles as well as high rate capabilities $(>2$ $\times 10^{7} \mathrm{~cm}^{-2} \mathrm{~s}^{-1}$ ). They conclude that the MSPC is a good candidate for tracking at high luminosity colliders.

The present article will mainly be devoted to $x$-ray applications of the MSPC. It will be demonstrated that, because of its high counting-rate capability, the MSPC will find many interesting applications, among them being an interesting device for synchrotron radiation research.

\section{MICROSTRIP PLATE DESIGNS}

The photolithographic technique allows electrodes to be precisely fabricated, thus permitting small electrode spacings with an accuracy and uniformity which cannot be achieved using wire-plane components. MS plates with an anode-strip width of $2 \mu \mathrm{m}$ and an anode-strip pitch of 200 $\mu \mathrm{m}$ have now been produced. These dimensions are about a factor of 5 smaller than achievable for a wire chamber. Moreover, the spacing between the anodes and the cathode strips, which collect the positive ion charge of the avalanche, can be made much smaller $(<50 \mu \mathrm{m})$ than the spacing $(>1 \mathrm{~mm})$ between anode and cathode wire planes of the conventional wire chamber. This results in a much faster signal development for the MSPC. A MS structure which has been used extensively for development work at both ILL and DSRI is shown in Fig. 1. The narrow anode strips are located between the wider cathode strips. The dimensions defining the MS structure are given in the inset. The applied voltage difference provides an electric potential which alternates between each strip and causes gas amplification for the electrons produced in the counter gas volume above the MS. The ends of the strips have been smoothly rounded to avoid electric fields which are much higher than those between the strips. Even with this precaution, however, the usable gas gain is limited. The gain limit for the MS structure shown in Fig. 1 was investigated by Budtz-J $\phi r g e n s e n ~ e t ~ a l .^{6}$ for different $\mathrm{Ar} / \mathrm{Xe}$ mixtures. The upper curve of Fig. 2 displays the gain limit below which the MSPC could be operated without sporadic electrical discharges. When the gain was increased above this limit, sparking took place at the circular endpoints of the anode strips. Investigations with a microscope showed that the sparks resulted in small tracks. A few sparks at the endpoints can be tolerated without any fatal effect on the MSPC. Excessively high gains can, however, result in disruption of the anode strips. The two lower curves in Fig. 1 show the gain as function of the Xe concentration for two different voltages between the anode and cathode strips. The relatively low voltage $(<1 \mathrm{kV})$ required to reach gains $>10^{3}$ is an advantage especially for space applications. 


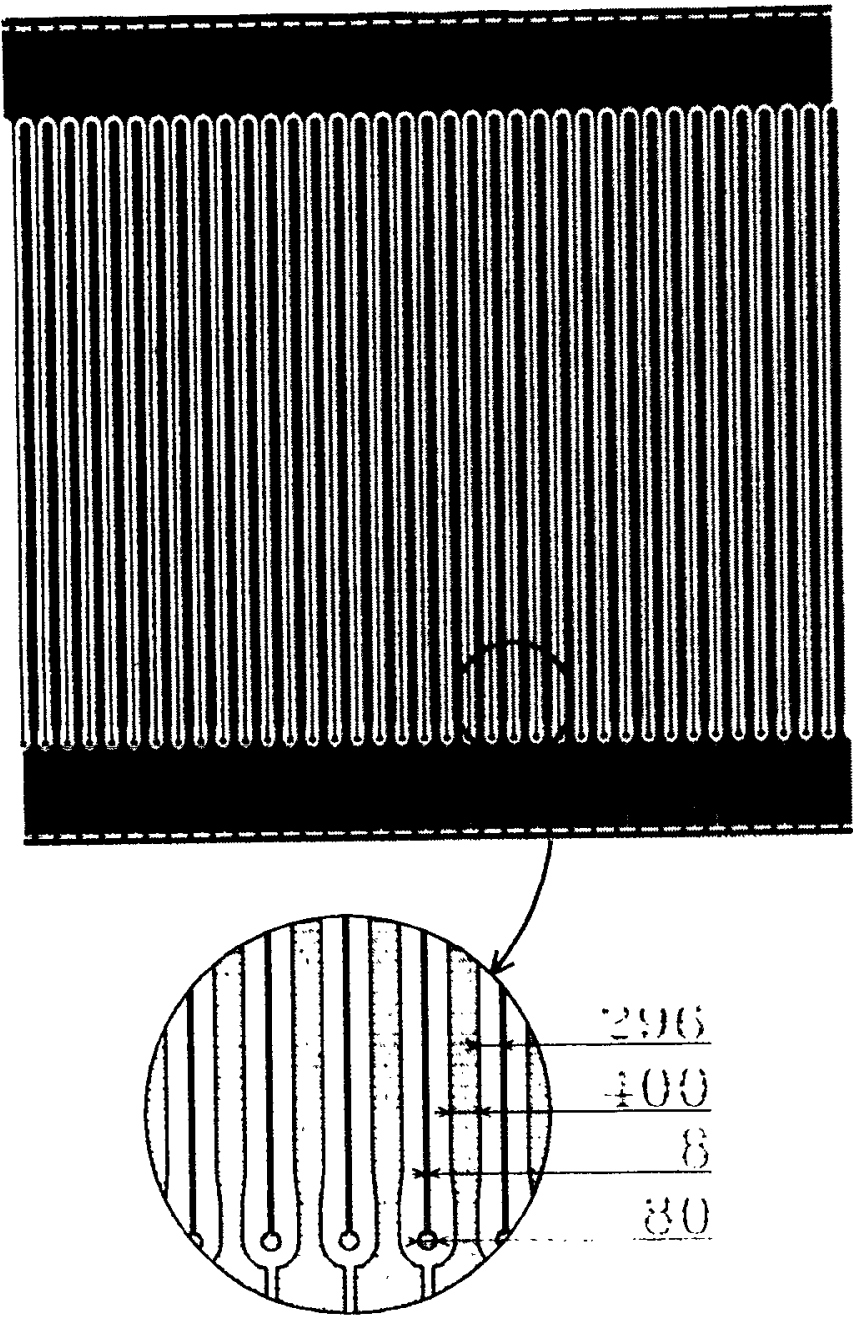

FIG. 1. Layout of the MS plate showing rounded strip ends. The dimensions are given in $\mu \mathrm{m}$. The $8-\mu \mathrm{m}$ strips are the anode.

A fundamental problem for the operation of the MSPC became apparent during the test of the early MS plates. ${ }^{7}$ Some of these plates showed strong gain variations as function of time. In addition, gain was dependent also on the counting rate. Investigations of these variations showed that they depend strongly on the type of glass used for the substrate. For quartz, which is an extremely good insulator, it was impossible to obtain a pulse-height spectrum because the time variation of the gain was faster than the time required to record one spectrum. Other glasses with lower resistivities, e.g., crown glass or green glass, allow acceptable energy spectra, but here were still gain drifts of up to $50 \%$ per $\mathrm{h}$. Some glasses showed gains which decreased with time after $\mathrm{HV}$ was switched on, while others had gains that increased. The gain drifts also depend on the dimensions of the MS structure. They can be reduced by depositing an electrode which is held at a positive potential with respect to the cathode strips on the backside of the MS plate.

Some of these observations may be explained by assuming that charge collects on the glass surface between the electrodes. If this surface is a sufficiently good insula-

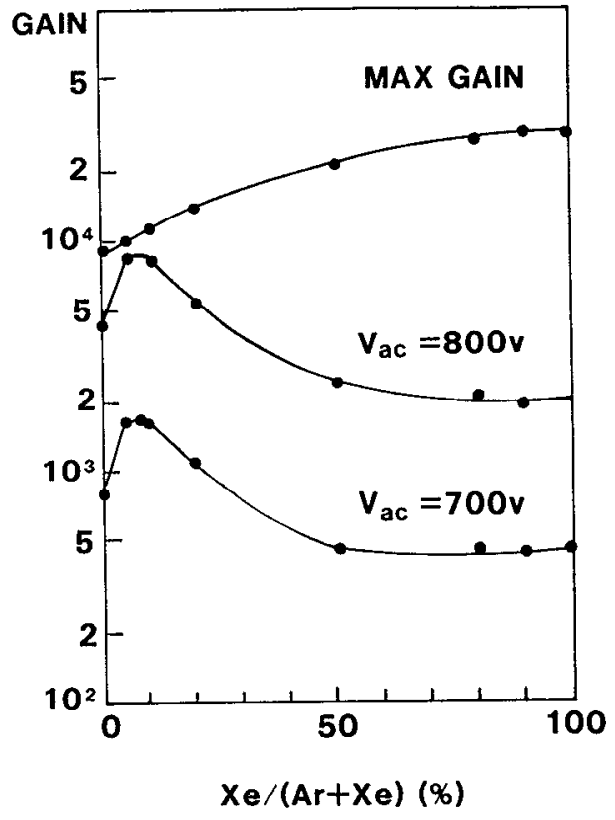

FIG. 2. Gas gain as function of Xe/Ar mixing ratio. All mixtures contain $10 \% \mathrm{CH}_{4}$. The two lower curves show the gain for voltages of 700 and $800 \mathrm{~V}$ between anodes and cathodes, respectively. The upper curve shows the highest gain at which the MSPC can be safely operated.

tor, then the charges accumulate and cause the amplifying electric field to be changed. The use of insulators with relatively low specific resistivity such that the charges reaching the surface are quickly removed will, therefore, cure the drift problem. Several "low" resistivity glasses were tested and one type, Corning 7740, a borosilicate glass with a $4,4 \% \mathrm{NaO}_{2}$ content, was found to be ideally suited as MS substrate. Its specific volume resistivity is $10^{8} \Omega \mathrm{cm}$ at $250^{\circ} \mathrm{C}$ which is relatively low compared with other $\mathrm{Si}$ based glasses. ${ }^{8}$ For the MS structure shown in Fig. 1, this glass resulted in gain drifts of only $\pm 1 \%$ over a one week period. The dependence on counting rate will be discussed later.

An alternative solution in which the surface conductivity of the MS plates is increased by means of ion implantation has also been tested. ${ }^{7}$ A quartz MS plate was doped with $2 \times 10^{15}$ phosphorus atoms $/ \mathrm{cm}^{2}$ showed a marked improvement over the pure quartz plate, which became charged immediately. The doped surface, however, yielded spectra of nearly the same quality as for the Corning 7740 glass. Further investigations are required in order to determine the optimal implantation dose.

Different kinds of material have been used for the electrode structures. Chromium layers of $0.1-0.2 \mu \mathrm{m}$ thickness are commonly used, since they adhere extremely well to the glass substrate. The drawback is that the $\mathrm{Cr}$ strips, especially the narrow anode strips which have rather high electrical resistivity, lead to a degradation of the risetime of the fast avalanche signals. MS plates produced by the socalled "lift-off-technique," which allows thicker MS structures to be produced without problems caused by under etching, ${ }^{9}$ avoid this problem. In this process, the metal layer is deposited on a substrate which is already covered 


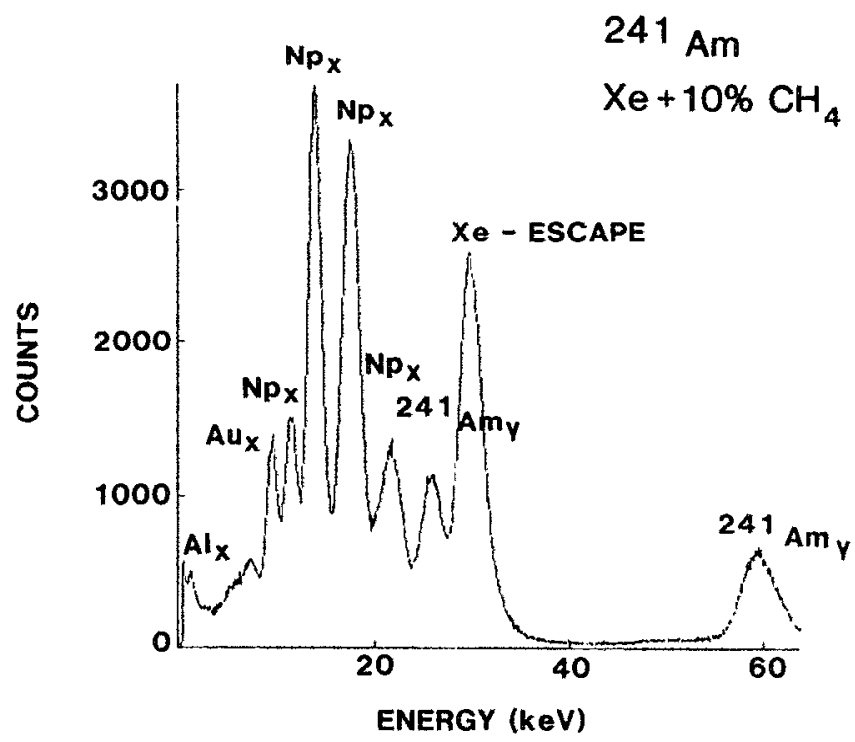

FIG. 3. MSPC pulse-height spectra obtained with a ${ }^{241}$ Am source.

with a negative resist image of the structure. Using a solvent, the resist is then removed together with the metal on its surface, leaving a positive metal image on the substrate. MS plates with layers of $1-\mu \mathrm{m}$-thick Al can be made, and they have nearly two orders of magnitude higher conductivity than the Cr plates. At first the Al plates had the same good energy response as the $\mathrm{Cr}$ plates, but they also showed time-dependent gain variations as discussed above. It is possible that oxidation of the $\mathrm{Al}$ surface forms insulating layers on the strips which then can result in a charge buildup, which affects the gain of the MS plate. This problem was solved when Au was used as conducting material. Since $\mathrm{Au}$ does not adhere well to glass substrate, the $\mathrm{Au}$ strips are deposited on an intermediate layer of $0.03-\mu \mathrm{m}$ - thick Ni. MS structures of 0.1 and $0.2 \mu \mathrm{m}$ Au with sufficient conductivity for our purposes were produced by the Lift-off-technique. ${ }^{9}$

\section{ENERGY RESPONSE}

The energy response of the MSPC to $x$ rays has been studied in some detail, especially for Ar- and Xe-based counter gas mixtures. ${ }^{3,4,10,11}$ Angelini et al. ${ }^{4}$ and BudtzJørgensen et $a l^{3}$ found energy resolutions $<13 \%$ (FWHM) in Ar mixtures for ${ }^{55} \mathrm{Mn}(5.9 \mathrm{keV}) \mathrm{x}$ rays, while Xe-based mixtures yield slightly increased values $\sim 13.5 \%$ (FWHM) ${ }^{10}$ It is remarkable that the energy resolution of the MSPC, in contrast with wire chambers, is nearly independent of gas gain, e.g., for $\mathrm{Xe}+10 \% \mathrm{CH}_{4}$ the resolution increases from $13.6 \%$ to $14.0 \%$ as the gain is increased from $\sim 10^{2}$ to $2 \times 10^{4}{ }^{10}$ The energy response of the MSPC is illustrated in Fig. 3, which shows energy spectrum obtained from an ${ }^{241} \mathrm{Am}$ source. The detector, which has an active area of $40 \times 30 \mathrm{~mm}^{2}$ and a $20-\mathrm{mm}$-deep drift region, was filled with a Xe $+10 \% \mathrm{CH}_{4}$ mixture at NTP. The drift field was $250 \mathrm{~V} / \mathrm{cm}$ and the MS plate was operated at a gain of $10^{3}$. The energy/pulse height relation is linear in the energy range investigated.

The low energy crystal spectrometer ${ }^{12}$ at DSRI was used in the energy range from 0.18 to $1.8 \mathrm{keV} .{ }^{11}$ The DSRI facility consists of a vacuum tank containing an $x$-ray generator and a crystal spectrometer, which selects the desired line energy by Bragg reflection. Two W/Si multilayer crystals with $2 d$ spacings of 100 and $54 \AA$, respectively, were used in the crystal spectrometer. The detector was equipped with a $1 \mu \mathrm{m}$-thick polyimide window coated with a $40-\mathrm{nm}$ layer of $\mathrm{Al}^{13}$ with a combined transmission $>10 \%$ at the energies investigated. The detector was operated with $\mathrm{Xe}+10 \% \mathrm{CH}_{4}$ as described above, except that the gas gain was raised to $\sim 10^{4}$. Figure 4 shows the MSPC pulse-height spectra for the $\mathrm{B} K, \mathrm{O} K, \mathrm{Ni} L$, and $\mathrm{Si} K$

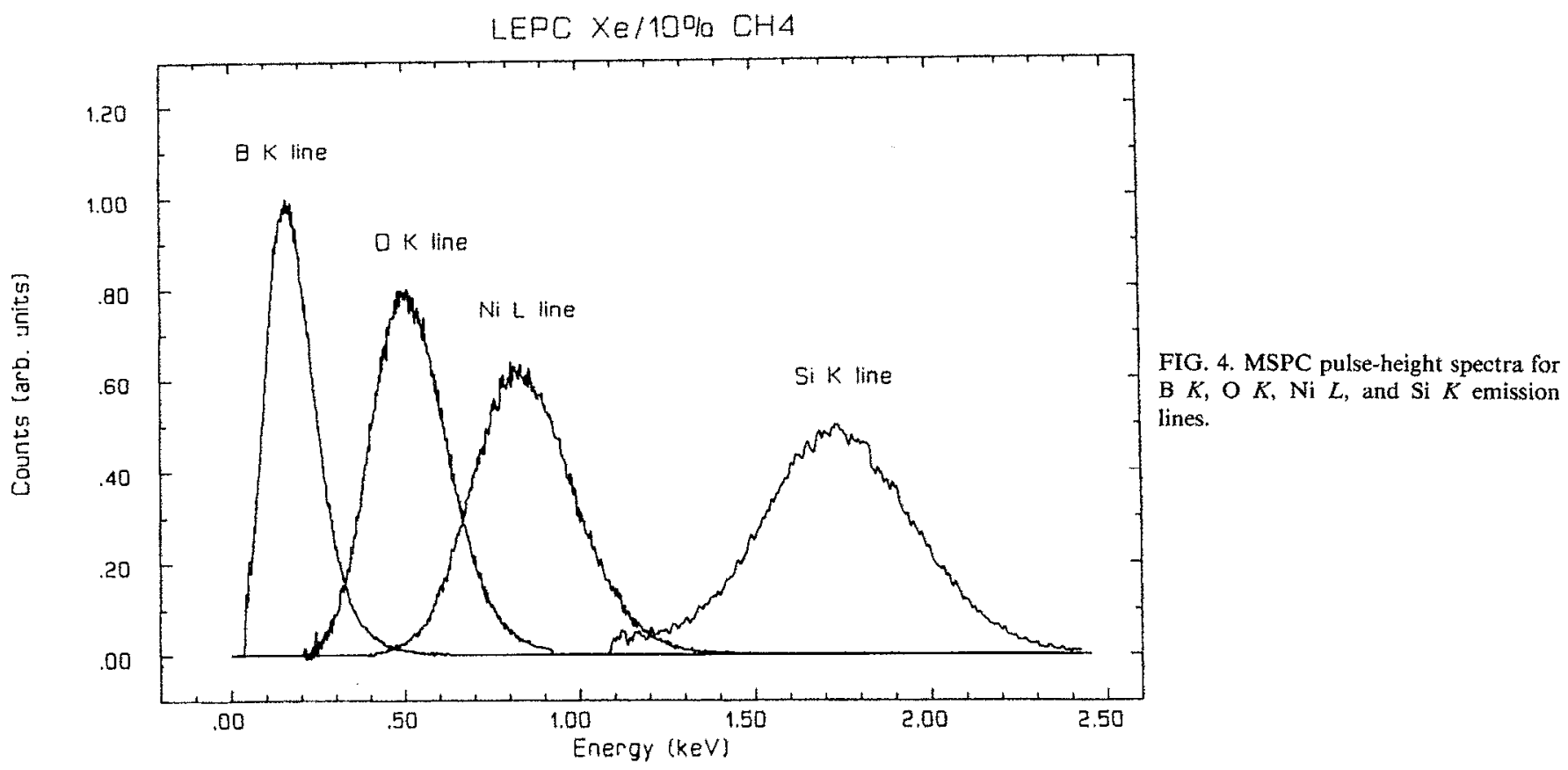




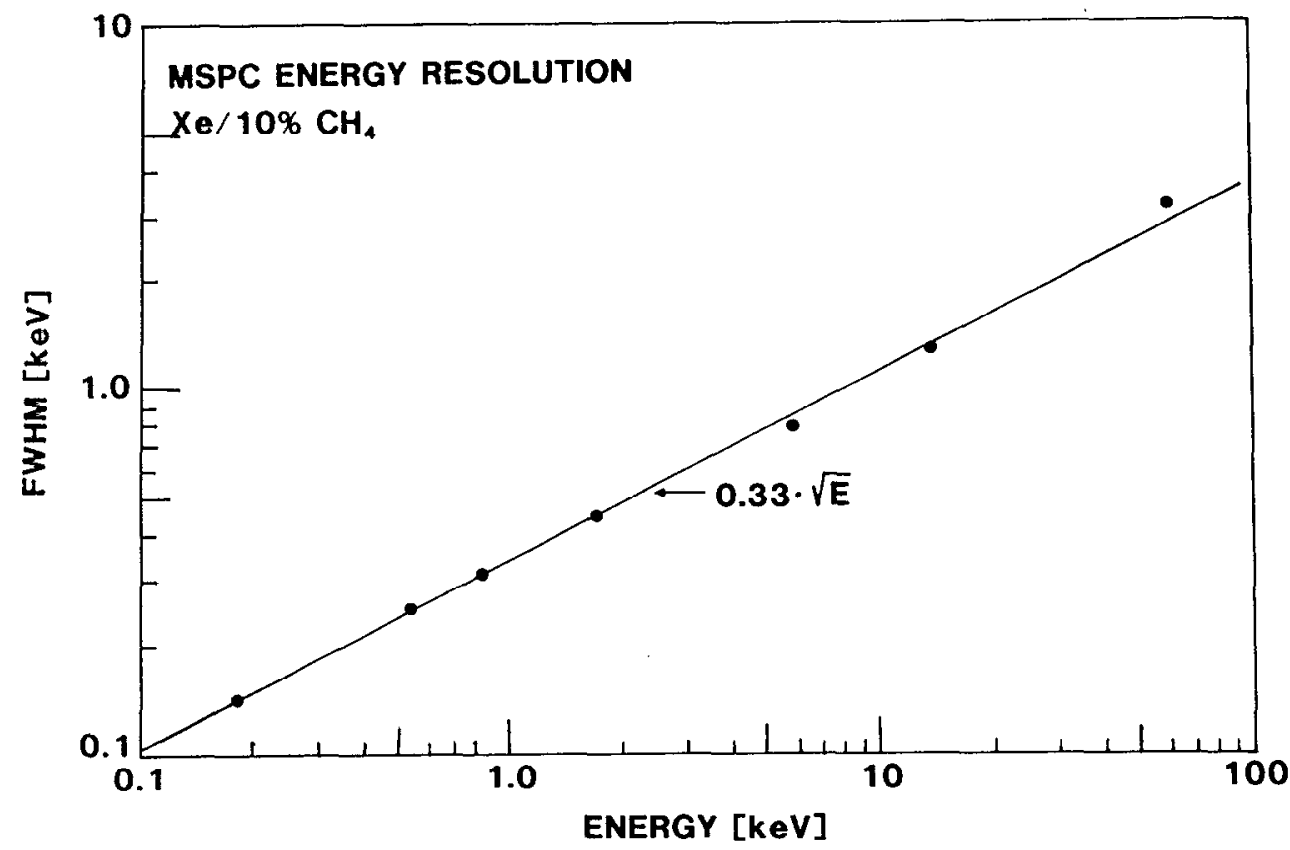

FIG. 5. The points indicate the measured MSPC energy resolution as function of the $x$-ray energy. The solid line indicates the square root of energy dependence.

emission lines at energies of $185,523,849$, and $1741 \mathrm{eV}$, respectively. Observe that the pulse-height spectra for the lowest lines are skewed with a high energy tailing. This is a consequence of the small number $(\sim 8$ for $\mathrm{B} K)$ of primary electrons produced in the gas. The energy resolutions (FWHM) measured in the $x$-ray energy range from 0.18 to $60 \mathrm{keV}$ are plotted in Fig. 5. The resolution varies as the square root of the energy with a proportionality factor of 0.33 :

$$
\Delta E(\mathrm{FWHM})=0.33 \times(E / 1 \mathrm{keV})^{1 / 2} \mathrm{keV} .
$$

\section{POSITION DETERMINATION}

An important advantage of the MSPC is that the strip pitch can be matched to the desired spatial resolution, i.e., the electrode distances can be chosen much smaller than for the MWPC, where the wire spacing for practical reasons is limited to $\geqslant 1 \mathrm{~mm}$. Differential nonlinearities arising from irregularities in the anode pitch in the MWPC can, therefore, be avoided. For a sufficiently fine pitch, the primary electrons produced by the incoming radiation will be distributed over more than one anode strip. An individual strip readout together with a determination of the centroid yields a very accurate position determination. Hartjes et $a l .^{5}$ achieved position determination in the direction orthogonal to the microstrips with accuracies in the range of $10 \mu \mathrm{m}$. An alternative method is to connect the anode (or cathode) strips to a common resistive stripline deposited on the MS plate. Localization is then achieved using conventional charge division. This is the method adapted for the DSRI imaging detectors developed for $x$-ray astronomy. ${ }^{10}$ The event localization in the direction parallel to the microstrips can be accomplished with electrodes deposited either below or above the MS plate which detect the charge induced by the avalanche. MS plates with strip electrodes on the backside of the substrate are rather easy to fabricate. Sufficient coupling can be achieved for thin substrates, e.g., the MS structure shown in Fig. 1 yields a coupling of $20 \%$ for $0.5-\mathrm{mm}$-thick substrates. The feasibility of the method was demonstrated by Budtz-Jørgensen et al. ${ }^{3}$ The position spectrum shown in Fig. 6 was recorded using a well collimated ${ }^{55} \mathrm{Fe}$ x-ray source.
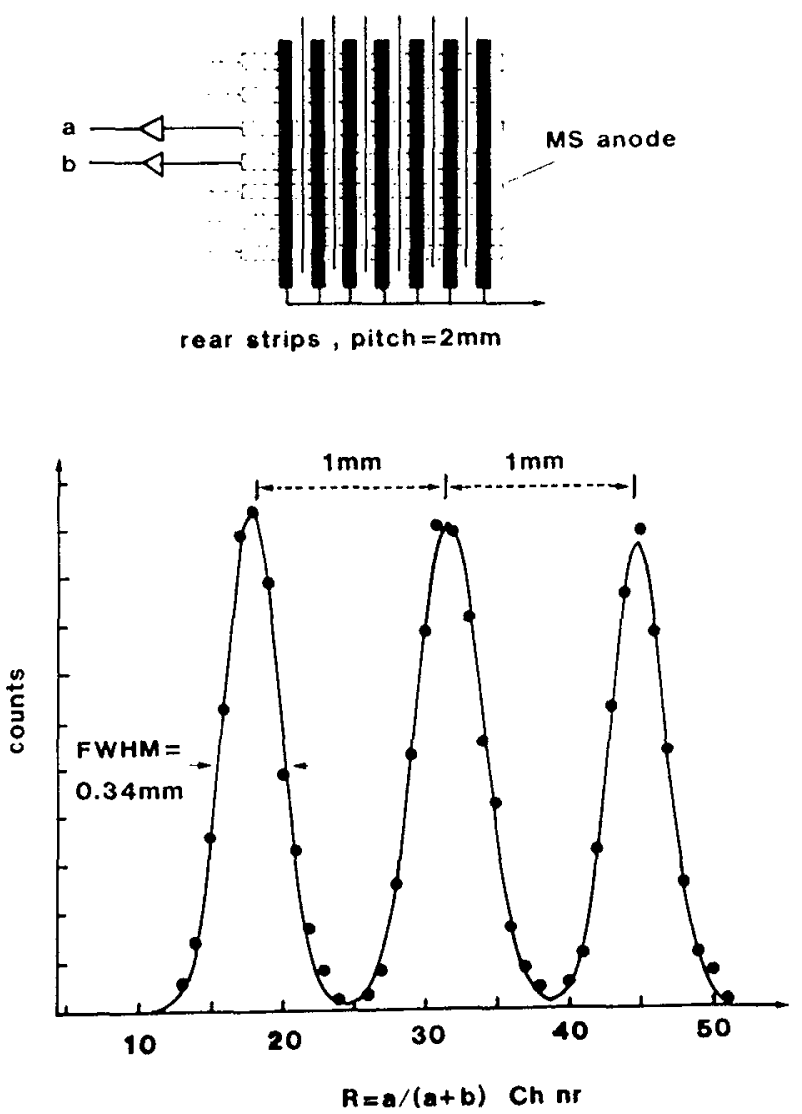

FIG. 6. Position determination using pick-up strips on the rear side (dashed) of the glass substrate. 
This readout has not been developed further at DSRI since the individual strip readout demands many more electronic channels than can be provided for in a spaceborne instrument. Instead, the signal induced in a resistively coupled wire grid placed above the MS plate is detected. Although this technique is more complicated mechanically, it yields a better signal-to-noise ratio and, therefore, the number of readout channels can be reduced, see Sec. VI.

\section{COUNTING RATE PERFORMANCE}

One of the most important features of the MSPC is its high counting rate capability, which is due partly to its fine pitch, which spreads the incoming flux over many electrodes, and partly to the very short ion collection time, which sharply reduces the space-charge buildup. Angelini et $a l^{4}{ }^{4}$ reported a rate capability $>10^{5}$ counts $\mathrm{s}^{-1} \mathrm{~mm}^{-2}$ for $8 \mathrm{keV} \times$ rays for a MS with an anode pitch of $0.2 \mathrm{~mm}$. A MSPC developed at DSRI was illuminated for $24 \mathrm{~h}$ by an intense ${ }^{55} \mathrm{Fe} \mathrm{x}$-ray source, which gave $1.2 \times 10^{6}$ ph s${ }^{-1}$ over an area of $1 \mathrm{~cm}^{2} .{ }^{10}$ The gain and the measured counting rate were stable during the illumination, and the resolution was unchanged after the exposure. A further investigation of the counting rate behavior of the MSPC was made with the DSRI rotating anode $x$-ray facility. The detector filled with $\mathrm{Ar}+10 \% \mathrm{CH}_{4}$ and operated at a gain of $10^{3}$ was exposed to an intense beam of $\mathrm{Cu} K(8 \mathrm{keV}) \mathrm{x}$ rays. The beam was collimated to a size of $0.5 \times 0.5$ $\mathrm{mm}^{2}$. The $\mathrm{x}$-ray tube was operated at $30 \mathrm{keV}$ and the tube current was varied from 20 to $180 \mathrm{~mA}$ in steps of $10 \mathrm{~mA}$. The MS plate produced energy spectra with a clean $\mathrm{Cu} K$ and Ar escape peaks, even for the highest counting rate of $\sim 5 \times 10^{5} \mathrm{ph} \mathrm{s}^{-1} \mathrm{~mm}^{-2}$. Some of the recorded spectra are shown in Fig. 7. A small gain drop was, however, observed with increasing counting rate. The decrease $(\Delta G)$ of the gain is linearly dependent on the counting rate $(R)$ such that

$$
\Delta G / G_{0}=C_{G} \times \mathrm{R} \text { with } C_{G}=0.4 \times 10^{-6} \mathrm{~s} \mathrm{~mm}^{2} .
$$

The cause of this gain behavior is not understood at present. If it is due to space-charge effects, then MS plates with finer strip pitch ( $<1 \mathrm{~mm}$ ) should be able to tolerate even higher counting rates. The counting rate limitations implied by Eq. (2) will not affect the performance of the DSRI MSPC, since even the brightest celestial $x$-ray source, SCO $x-1$, will give counting rates $<5 \times 10^{3} \mathrm{~s}^{-1}$ $\mathrm{mm}^{-2}$.

For applications in more intense radiation environments, such as synchrotron radiation facilities, the present results suggest that the MSPC can handle rates of $\sim 5$ $\times 10^{5} \mathrm{ph} \mathrm{s}^{-1} \mathrm{~mm}^{-2}$ without severe degradation of the pulse-height response. This is 1-2 orders of magnitude higher than for the MWPC. ${ }^{14}$

\section{PRACTICAL MSPC DESIGNS}

At ILL, Grenoble, Oed and Geltenbort are constructing a thermal neutron detector with a very large sensitive area with dimensions $400 \times 14 \mathrm{~cm}^{2}$. The detector will be
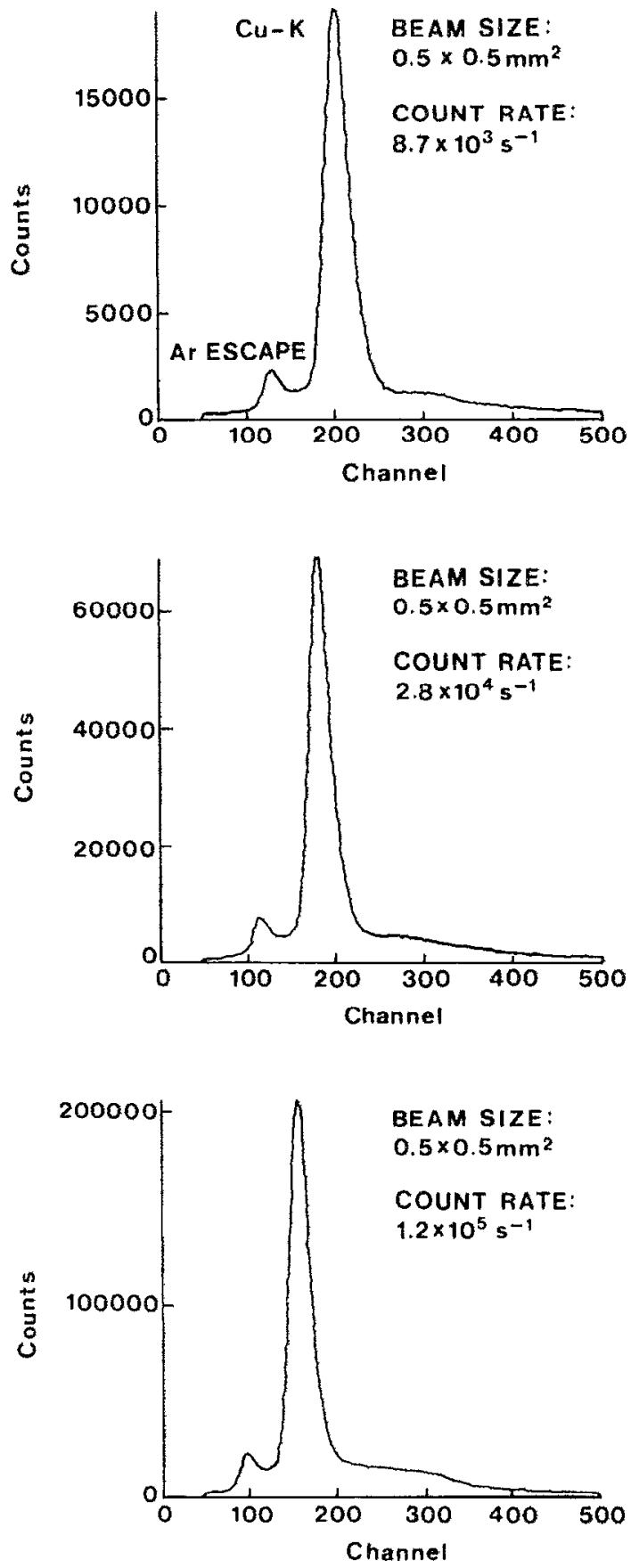

FIG. 7. Pulse-height spectra obtained with the rotating anode x-ray generator for three different beam intensities.

equipped with $50 \mathrm{MS}$ plates of each $168 \times 81 \mathrm{~mm}^{2}$. Each plate is divided into 32 cells of 2.54-rmm width having 6 microstrip structures. The detector has one-dimensional position sensitivity, which is achieved by a direct readout of the 1600 individual cells. The detector will be operated with a gas mixture of ${ }^{3} \mathrm{He}+25 \% \mathrm{CF}_{4}$ at 4 bars. Each cell will be able to handle rates of $\sim 10^{5}$ neutrons $\mathrm{s}^{-1}$.

DSRI will provide a set of four imaging MSPCs as part of XSPECT, the Danish contribution to two SODART $\mathrm{x}$-ray telescopes, which will be flown on the Soviet satellite SPECTRUM-RÖNTGEN-GAMMA planned for launch 
TABLE I. HEPC, LEPC specifications.

\begin{tabular}{|c|c|c|c|}
\hline \multirow[t]{2}{*}{$\begin{array}{l}\text { Energy resolution } \\
\text { Background rejection } \\
\text { Time resolution } \\
\text { Count rate } \\
\text { Gas }\end{array}$} & $\begin{array}{l}0.32 \times \\
>99 \% \\
<5 \mu \mathrm{s} \\
<5 \times 1 \\
\times \operatorname{Xe} 90 \%\end{array}$ & $\begin{array}{l}-1 \mathrm{~mm}^{-2} \\
0 \%\end{array}$ & \\
\hline & & HEPC & LEPC \\
\hline Field of view & $\operatorname{arc} \min$ & 60 & 30 \\
\hline Diameter of active area & $\mathrm{mm}$ & 150 & 75 \\
\hline Diameter of window & $\mathrm{mm}$ & 140 & 70 \\
\hline Position resolution & $\mathrm{mm}$ & $<1$ & $<2$ \\
\hline Energy range & $\mathrm{keV}$ & $2-25$ & $0.2-8$ \\
\hline Gas thickness & $\mathrm{cm}$ & 4 & 4 \\
\hline Gas pressure & atm & 1 & 0.5 \\
\hline Window: polyimide & $\mu \mathrm{m}$ & 3 & 0.5 \\
\hline Al & $n \mathrm{~m}$ & 40 & 40 \\
\hline
\end{tabular}

in 1994. A high and low energy (HEPC and LEPC) detector will be placed in the focal plane of each of the SODART telescopes. The HEPC and LEPC design specifications are given in Table $\mathrm{I}$.

The working principle of the detectors is illustrated in Fig. 8. The central electrode contains the microstrip structure, which has the same basic dimensions as the MS plate shown in Fig. 1. The HEPC-MS plate has an octagonal shape and a diameter of $150 \mathrm{~mm}$, while the LEPC plate is quadratic with dimensions of $82 \times 82 \mathrm{~mm}^{2}$. Position readout is based on the resistive charge division, depicted in Fig. 8. The microstrip cathodes are used for one position coordinate and, for the other coordinate, a wire grid is used. It consists of $35 \mu \mathrm{m}$-thick gold-coated tungsten wires at a pitch of $1 \mathrm{~mm}$ suspended a small distance above the MS plate, and biased to collect a large fraction of the ions. Resistors are connected between each wire/cathode. The charge-division method allows a coarse/fine readout sys-

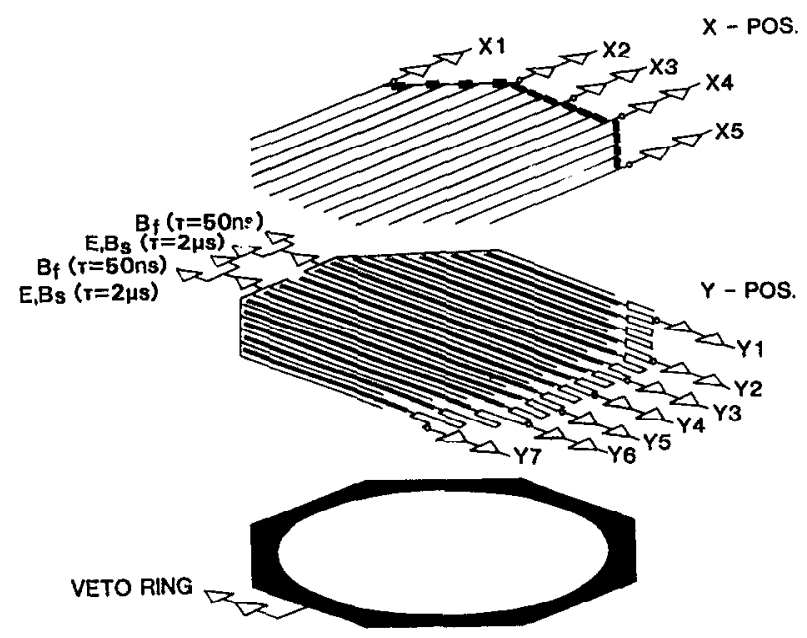

FIG. 8. The working principle of the XSPECT MSPC. The center plane shows the MS electrode. The anodes are subdivided into two separate sections. Each provides x-ray energy $(E)$ and two pulses $\left(B_{f}, B_{S}\right)$ obtained from amplifiers with different shaping time constants. The MS cathodes are connected with a resistive meander strip. The upper electrode is a wire grid for resistive readout in the $x$ direction. The lower electrode is a veto electrode surrounding the useful area. tem in which the resolution can be improved by a "simple" subdivision of the resistive chain. For the XSPECT MSPC, six subdivisions of the cathode plane and four of the grid plane are sufficient.

In space, the detectors will be exposed to a flux of charged particles, mainly protons and electrons, which produce detector events with pulse heights in the $\mathrm{x}$-ray range of interest. Charged particles differ from $\mathrm{X}$ rays with respect to their pulse shape and can, therefore, be rejected using pulse-shape analysis techniques. This method compares the fast rise time of $\mathrm{x}$-ray signals ( $50 \mathrm{~ns}$ in Ar, $100 \mathrm{~ns}$ in $\mathrm{Xe}$ ) with the slow rise time $(>1 \mu \mathrm{s})$ of pulses produced by charged particles crossing the 4-cm-deep detector volume. The output of the anode amplifier is fed in parallel to two shaping amplifiers with shaping times of $2 \mu$ s and $50 \mathrm{~ns}$ resulting in two pulses with pulse heights $B_{s}$ and $B_{f}$, respectively. The quantity $R=B_{f} /\left(B_{f}+B_{s}\right)$ is very sensitive to rise time and yields distinctly different values for $\mathrm{x}$-ray events than for charged particles crossing the detector.

Particles moving parallel or nearly parallel to the MS plate produce pulses which can have short rise times. They are, however, rejected by the signal they induce in the veto electrode (Fig. 8), which is mounted on the rear side of the MS plate, and which surrounds the useful window area. From laboratory measurements, it is estimated that charged particle events can be rejected with an overall efficiency of $99.5 \%$ using both rise-time and veto-rejection techniques.

The properties of the MSPC, especially the high rate capabilities, make this detector type an obvious candidate for use at synchrotron facilities. The required detector performance at synchrotrons is indeed extreme-local counting rates $\sim 10^{6} \mathrm{ph} \mathrm{s}^{-1}$ and overall counting rates $\sim 10^{7}-10^{9}$ ph s ${ }^{-1} \cdot{ }^{15}$ The possible application of the MSPC as a synchrotron detector is being investigated through a collaboration between the Daresbury Laboratory, UK, and DSRI. On the short term, this project will be devoted to the development of a one-dimensional $200-\mathrm{mm}$ aperture $\mathrm{x}$-ray detector using a gas microstrip and a fast parallel readout of the type developed at Daresbury. ${ }^{16} \mathrm{~A}$ schematic layout of the system is shown in Fig. 9. The detector is arranged with the direction of the strips perpendicular to the position-sensitive axis. Each anode strip is separately instrumented with an amplifier-discriminator-scaler combination such that one anode strip represents one pixel.

The outputs of the discriminators are counted by Daresbury 32 channel, fast camac scaler modules. These units are capable of operating at $180 \mathrm{MHz}$ per channel and set the maximum theoretical data rate. In practice the data rate will be set by the pulse pair resolution $(\sim 100$ ns for Ar) and space charge effects as discussed earlier. A uniform exposure counting rate in excess of $20 \mathrm{MHz}$ is predicted.

\section{CONCLUSION}

The MSPC has proven to be a major step forward in gas-detector technology. Almost all the characteristics of the detector (spatial resolution, energy resolution, rate ca- 


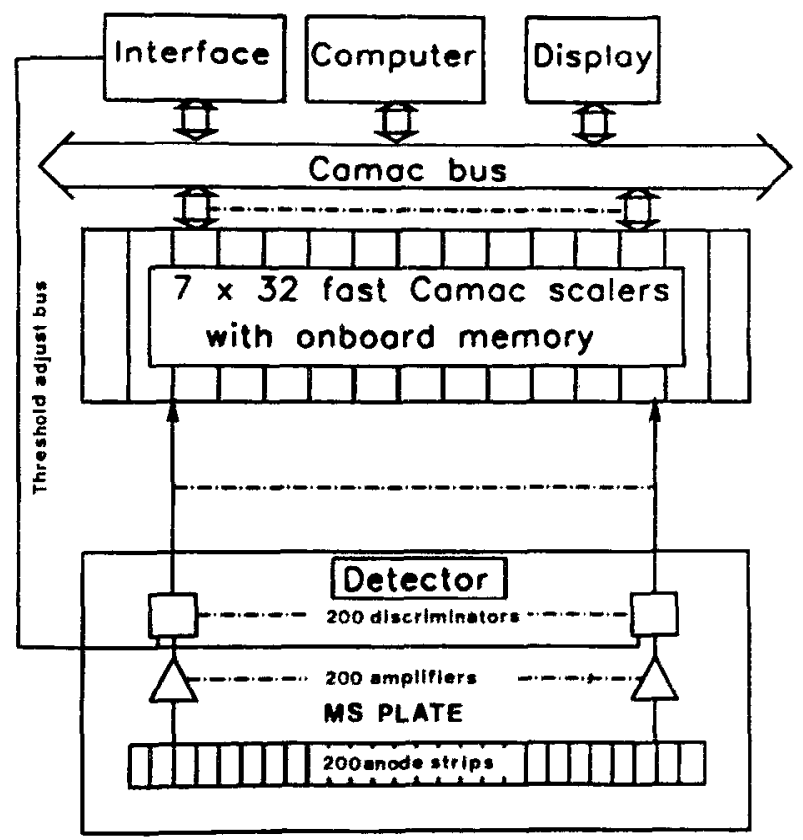

FIG. 9. Layout of the MS plate shown connected to the Daresbury fast parallel readout.

pability) are significantly improved over those of the standard MWPC. MSPCs are now being developed for use in a wide range of fields: Neutron diffraction, $x$-ray astronomy, high energy physics and synchrotron radiation. Lithographic technology permits a flexible and nearly optimal layout of the microstrip structures. A future coupling of modern microelectronics with the MS plate, as it is now the case for solid state strip detectors, will be a necessary step in achieving the full potential of the MSPC.

A. Oed, Nucl. Instrum. Methods A 263, 351 (1988).

${ }^{2}$ A. Oed, P. Convert, M. Berneron, H. Junk, C. Budtz-Iørgensen, M. M. Madsen, P. Jonasson, and H. W. Schnopper, Nucl. Instrum. Methods A 284, 223 (1989).

${ }^{3}$ C. Budtz-Jørgensen, M. M. Madsen, P. Jonasson, H. W. Schnopper, and A. Oed, SPIE Proc. 982, 152 (1988).

${ }^{4}$ F. Angelini and R. Bellazzini, Nucl. Instrum. Methods A 283, 755 (1989).

${ }^{5}$ F. Hartjes, B. Hendriksen, and F. Undo, Nucl. Instrum. Methods A 289, 384 (1990).

${ }^{6} \mathrm{C}$. Budtz-Jorgensen, A. Bahnsen, C. Olesen, M. M. Madsen, P. Jonasson, and H. W. Schnopper, Nucl, Instrum. Methods A 310, 82 (1991).

${ }^{7}$ C. Budtz-Jørgensen, M. M. Madsen, P. Jonasson, N. J. Westergaard, A. Bahnsen, and H. W. Schnopper, SPIE Proc. 1159, 236 (1989),

${ }^{8} \mathrm{D}$. Ans-Lax, Taschenbuch fur Chemiker und Physiker (Springer, Berlin, 1967).

${ }^{9}$ The MS plates were fabricated by Mettler-Toledo AG, 8606 Greifensee, Switzerland.

${ }^{10} \mathrm{C}$. Budtz-J $\phi r g e n s e n$, A. Bahnsen, C. Olesen, M. M. Madsen, P. Jonasson, and H. W. Schnopper, SPIE Proc. 1344, 91 (1990).

${ }^{11}$ C. Budtz-I . Madsen, P. Jonasson, and H. W. Schnopper, to be presented at SPIE Conference, 1991 (unpublished)

${ }^{12}$ F. E. Christensen, A. Hornstrup, P. Frederiksen, P. Grunds $\phi$, S. Henrichsen, E. Jacobsen, P. Jonasson, M. M. Madsen, C. Nilsson, H. W. Schnopper, N. J. Westergaard, and P. Đrup, J. X-ray Sci. Technol. 2, 81 (1990).

${ }^{13}$ S. Nenonen, H. Sipilä, P. Jalas, and R. Multikainen, SPIE Proc. 1344, 100 (1990).

${ }^{14}$ F. Angelini et al., Proceedings of ECFA Study Week on Instrument Technology for Highluminosity, Hadrou Colliders, CERN 89-10, 1989 (unpublished), p. 465.

${ }^{15} \mathrm{C}$. Riekel, Summary of ESRF detector workshop, Grenoble, 1990 (unpublished).

${ }^{16}$ R. Lewis, J. S. Worgan, N. S. Fare, I. L. Sunner, F. d'Annunzio, A. Berry, B. Parker, K. Roberts, and G. Mant, SPIE Proc. 1159, 205 (1991). 